\title{
Bistatic spaceborne-airborne experiment TerraSAR-X/F-SAR: data processing and results
}

\author{
Marc Rodríguez-Cassolà, Stefan V. Baumgartner, Gerhard Krieger, Anton Nottensteiner, Ralf Horn,
} Ulrich Steinbrecher, Robert Metzig, Markus Limbach, Pau Prats, Jens Fischer, Marco Schwerdt, Alberto Moreira

*Microwaves and Radar Institute, German Aerospace Center (DLR), 82234 Wessling, Germany

Phone/Fax: +498153282392/1449, Email: marc.rodriguez@dlr.de

\begin{abstract}
Following an original proposal by the authors to the TerraSAR-X (TSX) scientific coordination board, a spaceborneairborne bistatic experiment was successfully performed early November 2007. TSX was used as transmitter and DLR's new airborne radar system, F-SAR, as receiver; due to the capability of the latter to acquire data quasi-continuously, no echo window synchronisation is needed. Monostatic data were also recorded during the acquisition. This paper includes description and results of the spaceborne-airborne bistatic experiment, with special focus on data processing and image comparison. Given the acquisition scenario, with two-channel sampling and transmitter and receiver clocks operating independently, data processing must necessarily follow a three-step strategy: 1) channel balancing, 2) data synchronisation and 3) bistatic SAR processing. Since neither absolute range nor Doppler references are available in the bistatic data set, synchronisation is done with the help of calibration targets on ground and based on the analysis of the acquired data compared to expected data. Due to the variant nature of the bistatic acquisition and the required precision for the processing, data are processed using a bistatic backprojection approach.
\end{abstract}

\section{INTRODUCTION}

Several airborne bistatic SAR experiments [1], [2] have been successfully carried out since the end of 2002, showing very promising results and drastically sparking the interest of the scientific community on this topic. A new border in bistatic SAR campaigns has been recently crossed and results of a spaceborne-airborne acquisition are shown in the present paper. Proposed by the authors and accepted by TSX scientific coordination board in 2005, this first bistatic spaceborne-airborne experiment has been successfully conducted in November 2007 [3] involving TSX as transmitter and the new airborne system of DLR, F-SAR, as receiver, mainly due to its capability to acquire data quasi-continuously, which solves a major issue in bistatic data recording: echo window synchronisation. To the authors knowledge, this is the first civilian bistatic X-band experiment using a SAR satellite and an airborne platform, being the first including high-resolution SAR processing of the data. The bistatic experiment has been carried out during TSX commissioning phase to obtain a better characterisation of its transmit channel; it is also an important step in the preparation of TanDEM-X mission, including the verification of performance decrease caused by the independent use of two clocks [4] and the validation of processing and imaging techniques. The paper is divided in three different parts: a) presentation of the experiment and performance, b) data processing and c) results and image analysis.

\section{DESCRIPTION OF THE EXPERIMENT}

\section{A. Bistatic configuration}

Trajectories of both platforms are designed to be nearly parallel to maximise footprint overlapping; bistatic side-looking operation is preferred to simplify processing and avoid poor range resolution configurations. F-SAR is flying at a height of $2180 \mathrm{~m}$ with a mean speed of $90 \mathrm{~m} / \mathrm{s}$; TSX flies on a 514 $\mathrm{km}$ orbit with orbital speed of $7600 \mathrm{~m} / \mathrm{s}$. Figure 1 illustrates the geometry of the bistatic TSX/F-SAR acquisition. The nominal mid-beam incidence angles are $55.63^{\circ}$ for TSX and $45^{\circ}$ for F-SAR. The bistatic experiment has been performed near Kaufbeuren airfield, a present calibration test site for DLR Microwaves and Radar Institute; in order to avoid conflict with other TSX acquisitions over Germany, the satellite is specially operated in left-looking mode.

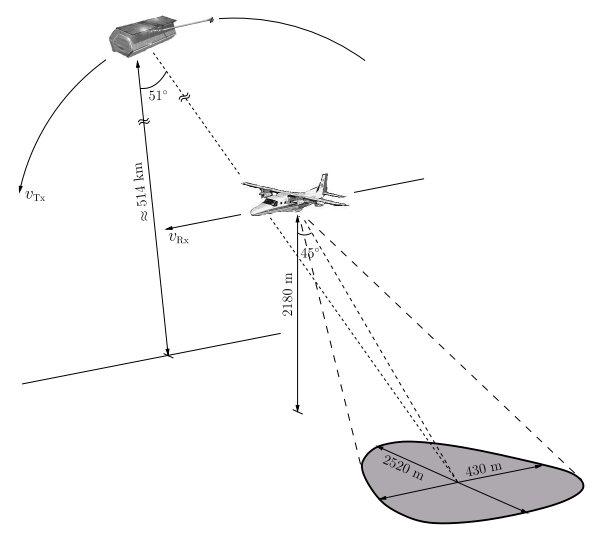

Fig. 1. Bistatic configuration in spaceborne-airborne TSX/F-SAR experiment.

\section{B. Bistatic acquisition}

Continuous bistatic data acquisition is preferred to overcome echo window synchronisation problems; however, the duty cycle of receiver ADCs must be kept under $50 \%$ and two ADCs must be switched to enable continuous data recording [3]. To avoid hardware modifications conflicting with the then development state of F-SAR X-band receiver chain, two 
different receiver channels have been used. Switching between both channels gives a sampling blank area of $1.6 \mu \mathrm{s}$ and is performed with a $7693.25 \mathrm{~Hz}$ cadence; maximal real sampling rate using this configuration is $250 \mathrm{MHz}$. Selected transmitted chirp bandwidth is $100 \mathrm{MHz}$ and down converting reference in receiver matches the nominal value of TSX carrier for this acquisition, $9.65 \mathrm{GHz}$. Chirp duration is $33.189 \mu \mathrm{s}$. To increase footprint overlapping time, TSX antenna is steered in azimuth to perform a spotlight illumination of the scene. F-SAR is receiving in regular stripmap mode. A high PRF of $5920.59 \mathrm{~Hz}$ is chosen to guarantee a high along-track oversampling rate of the bistatic data. Since the imaged scene is limited by F-SAR antenna pattern, no range ambiguities are expected to arise in bistatic image; range ambiguities are however expected in monostatic image, even worsened by the atypically shallow look angle used in the satellite antenna pattern. Transmitted peak power is $2.01 \mathrm{~kW}$. Taking into account the difference in transmitted power, antennas and free space losses of this bistatic acquisition compared to a pure airborne monostatic one, F-SAR receiver gain has been increased $22 \mathrm{~dB}$ with respect to monostatic operation. Three X-band transponders used for TSX calibration during its comissioning phase are used as reference targets on ground. Their exact position is measured with GPS; these targets will be essential to synchronise the data in further processing steps.

\section{Imaged scene}

Dimensions of TSX antenna are $4.784 \mathrm{~m}$ azimuth and 0.7 $\mathrm{m}$ elevation. F-SAR X-band antenna is smaller and has $0.2 \mathrm{~m}$ azimuth and $0.046 \mathrm{~m}$ elevation. TSX aperture is thus larger, but footprint on ground is logically much wider, given the correspondent slant ranges. Bistatic footprint thus coincides with F-SAR footprint during the time TSX illuminates the scene. Within the 3-dB part of F-SAR antenna, the ground range dimension of the imaged scene is $2520 \mathrm{~m}$; assuming footprint overlapping occurs for three seconds, along-track size of the scene varies between 621 and $878 \mathrm{~m}$ for near and far range, respectively. Footprint overlapping time is limited by standard duration of TSX high-resolution spotlight acquisitions. Bistatic data have been recorded during approximately $3.5 \mathrm{~s}$.

\section{Performance estimation}

For a computation of the expected performance of this bistatic system, spotlight processing of the whole scene will be considered. Using the formula presented in [5], the expected cross-track resolution projected on ground range varies within the 3 -dB part of F-SAR elevation beam between 2.2 and 1.7 $\mathrm{m}$ for near and far range, respectively. An analogous formula for along-track resolution also from [5] yields values between 0.25 and $0.39 \mathrm{~m}$ for $2.77 \mathrm{~s}$ integration time, whereas the value of the monostatic acquisition remains almost constant around $0.95 \mathrm{~m}$. SNR for the bistatic image is expected to be much higher than for the monostatic image, due to the much shorter receiver path in the bistatic acquisition. NESZ values for scene center are of $-40 \mathrm{~dB}$, having a $20 \mathrm{~dB}$ advantage over the monostatic image.

\section{DATA PROCESSING}

Data processing of this bistatic acquisition follows a three step approach: a) channel balancing, b) data synchronisation and c) focussing. Channel balancing is necessary due to the need of coherently combining data acquired with two different channels. The second step, data synchronisation is split into two steps: in the first one, nominal TSX PRF is used to create the bistatic data matrix; in the second one, precise frequency offset between TSX and F-SAR is estimated and data can be finely synchronised. The last step is regular bistatic SAR focussing. Figure 2 shows a block diagram of the different processing blocks and a corresponding data plot after each step.

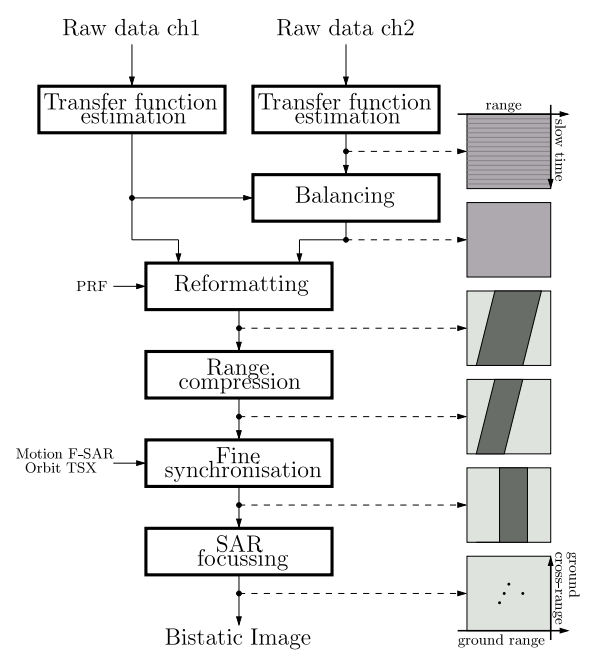

Fig. 2. Block diagram of processing for TSX/F-SAR bistatic acquisition.

\section{A. Channel balancing}

The use of two different receiver channels switched at a higher frequency than TSX PRF induces a frequent almost pulse-to-pulse modulation on the bistatic data. The origin of this modulation are: a) different DC offsets, b) different transfer functions and c) different electrical paths. The first two can be averaged using the raw data; the third is estimated by computing the signal energy found in adjacent ghost frequency bands after correcting with a constant sweeping phase for one of the channels. Energy maximum in central band coincides with energy minima in adjacent bands, and vice versa. Phase offset between both channels has been found to be $227.5^{\circ}$, which corresponds to an uncalibrated delay of $\left(65.49+k / f_{0}\right)$ ps, being $k$ an integer accounting for the ambiguity in the phase measure and $f_{0}$ the carrier frequency of transmitter.

\section{B. Coarse data synchronisation}

The nominal PRF of TSX yields a range line dimension of 21113 samples. However, these reformatted raw data clearly lack a precise range synchronisation, essential to guarantee high-resolution SAR imaging, even if Doppler synchronisation is guaranteed (which is neither the case). After this coarse range synchronisation, range compression (RC) and a quicklook are computed. The direct signal and several point targets 
are visible in RC data set. The effect of using, without proper a priori calibration, two different channels is shown in figure 3. Both images show the computed quick-looks for balanced bottom- and unbalanced -top- data sets. The presence of alongtrack ghosts is clearly visible in the unbalanced version of the quick-look image.

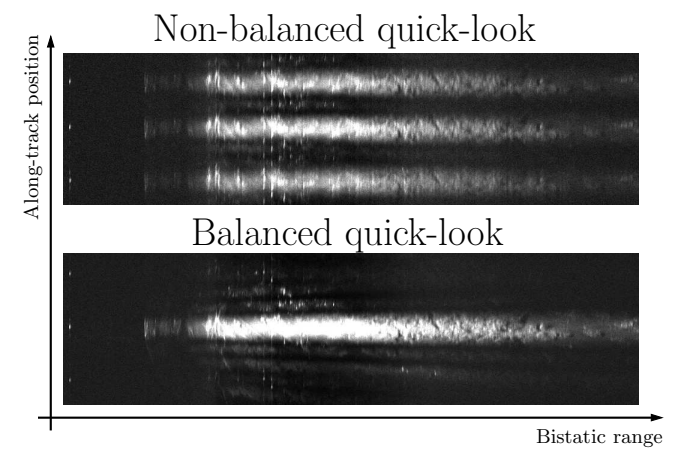

Fig. 3. Quick-looks for balanced -bottom- and non-balanced -top- data sets.

\section{Fine synchronisation}

Due to the short duration of the bistatic acquisition, no fast variation of clocks relative behaviour is expected in the bistatic data set. Thus only a constant offset in carrier frequency must be estimated. By inspecting the phase of direct signal and response of one transponder, carrier offset is estimated, within the chosen PRF, to be $2841.14 \mathrm{~Hz}$. Timing of the bistatic data set is then finely synchronised using a linear shift so that phase centre of direct signal coincides with its closest approach. PRF offset measured after this linear correction yields a value of $-0.01278715 \mathrm{~Hz}$, which scaled in carrier frequency converts into $-20.842 \mathrm{kHz}$, up to $0.5 \mathrm{~Hz}$ the same value obtained by unwrapping the previously estimated value.

\section{Focussing}

After precise data synchronisation, and due to the high resolution of the focussed image, focussing follows using a backprojection approach. A grid laid at a constant height on a spherical Earth is used as output image. No significant phase errors due to topography changes are expected since the whole scene is virtually flat. Residual phase error in direct signal and central transponder is kept under $\pi / 2 \mathrm{rad}$.

\section{RESUlts}

\section{A. Bistatic image}

Figure 4 shows the full bandwidth bistatic spotlight image without any pattern compensation. The image shows Kaufbeuren city and airfield, surrounded by forest and fields. Horizontal side correponds to ground range, from right (near) to left (far). The effect of F-SAR azimuth antenna pattern is clearly visible. On the farther side of the airfield track, the three X-band transponders can be seen. The interpolated response of the central transponder is plotted in figure 5 . Monostatic -black-, actual bistatic -red- and ideal bistatic blue- along-track responses are shown. Resolutions are 0.97 $\mathrm{m}, 0.53 \mathrm{~m}$ and $0.36 \mathrm{~m}$, respectively; the loss in resolution and increase of side lobes in the bistatic response is caused by uncompensated residual motion errors of F-SAR.

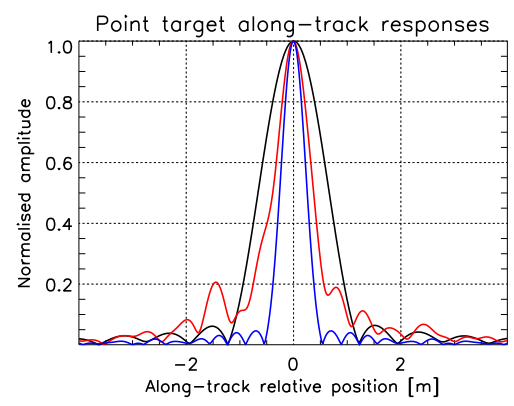

Fig. 5. Transponder response in monostatic -black-, bistatic -red- and ideal bistatic -blue-

\section{B. Monostatic vs. bistatic images}

In addition to the different resolutions expected in both images and the absence of range ambiguities in the bistatic image, four differences can be outlined in the bistatic vs. monostatic image comparison: a) SNR, b) geometrical mapping, c) backscattering mechanisms and d) Doppler information. Figure 6 shows a crop of bistatic and monostatic images giving an example of the SNR difference. The trackand-field facilities seen in bistatic -middle- and optical right- images are not visible in the monostatic image -left- Geometrical mapping and backscattering mechanisms are
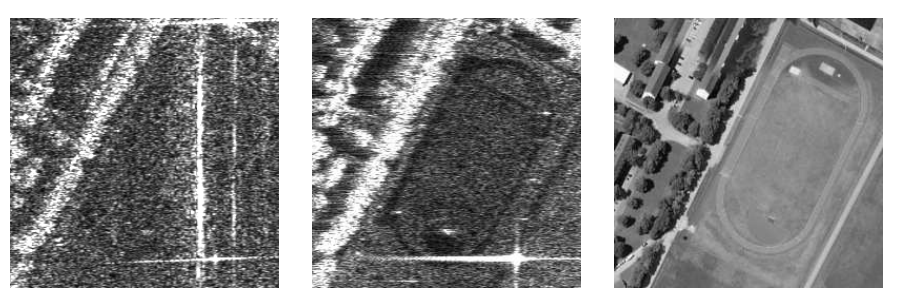

Fig. 6. Track-and-field facilities next to Kaufbeuren airfield. Crops of monostatic -left-, bistatic -middle- and optical -right- images. Note: the monostatic image does not achieve nominal TSX performance in terms of range ambiguity-to-signal ratio, due to the high PRF combined with large incidence angle.

a direct consequence of the different incidence angles seen by transmitter and receiver. For the presented image, TSX incidence angles remains almost constant around $55^{\circ}$, whereas F-SAR incidence angles vary from near to far range from $25^{\circ}$ to $68^{\circ}$. One example of the different geometrical mapping of both images is shown in figure 7. The image shows a crop of a forest near Kaufbeuren in monostatic -left- and bistatic -right- images. For the left part of both images, the shadows of the trees are clearly larger in the bistatic image. The incidence angle of receiver for this part of the image varies between $58.5^{\circ}$ and $64^{\circ}$; once again, the difference in radiometric resolution is noticeable. Another interesting crop accounting for the differences in monostatic and bistatic backscattering can be seen in figure 8. Again, monostatic -left, bistatic -middle- and optical -right- crops of the same area, some buildings near the airport, are shown. The buildings in 


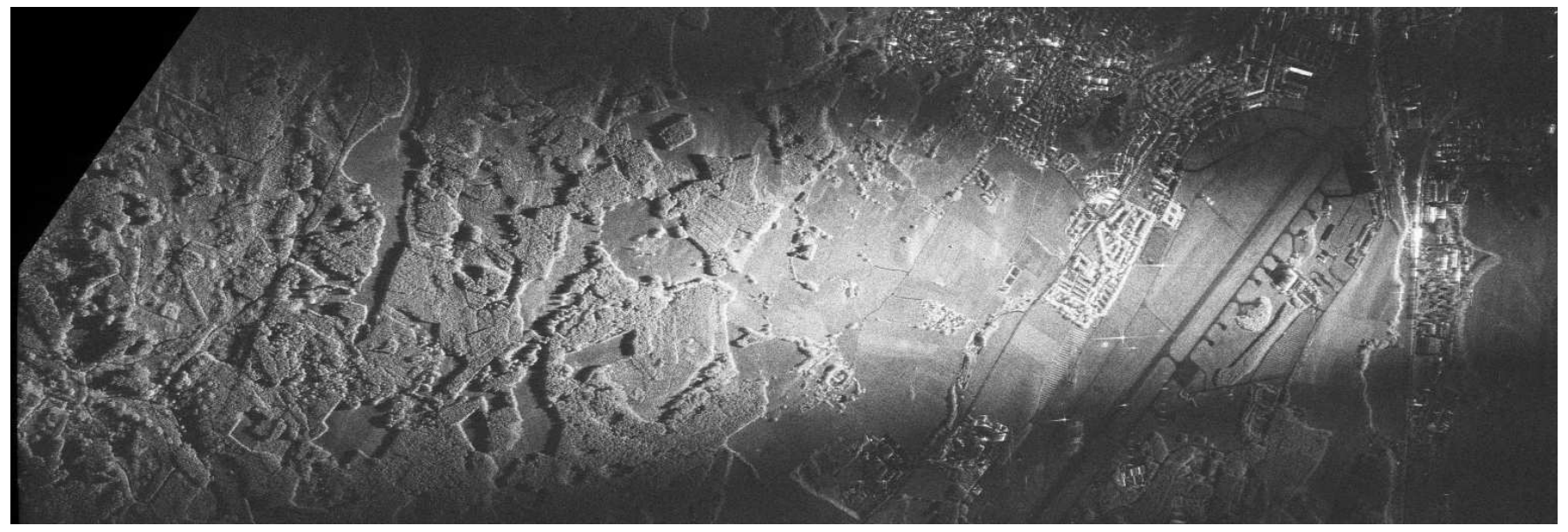

Fig. 4. Bistatic TSX/F-SAR complete image.

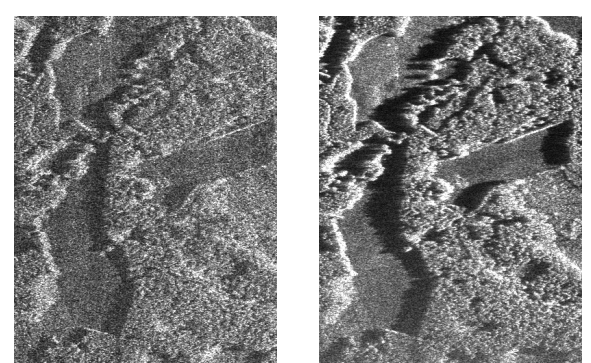

Fig. 7. Forest near Kaufbeuren. Crops of monostatic -left- and bistatic -rightimages.

the image appear brighter in the monostatic image. A second difference can be outlined between the two main groups of buildings, in what appears to be a metallic fence surrounding two buildings and the access road; this metallic fence, very bright in the monostatic crop, is completely invisible for the bistatic system. The third point can be seen on bottom of the images, with a solar panels field, also very bright in the monostatic image and much darker for the bistatic system. The
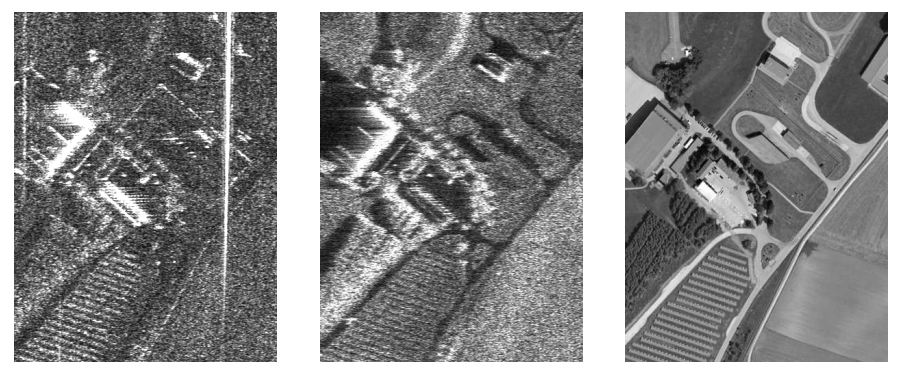

Fig. 8. Buildings and solar panels near airfield. Crops of monostatic -left-, bistatic -middle- and optical -right- images.

last crop -figure 9- of the image helps illustrate the differences in Doppler components of both images. The images show the three different transponders in the bistatic image. Due to the different spectral information of each transponder (caused by the variant character of the acquisition), the equivalent squint angles are different and the responses of first -top- and second -middle- transponders are no longer orthogonal.
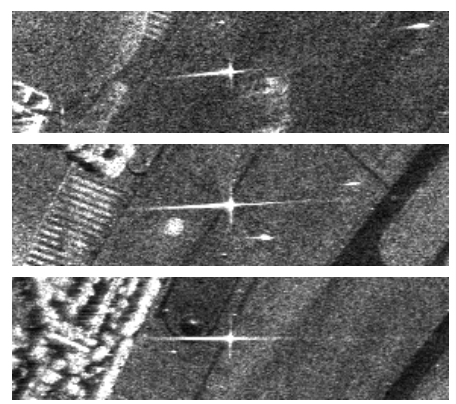

Fig. 9. Transponder responses in bistatic image.

\section{SUMMARY}

For the first time, a spaceborne-airborne X-band acquisition has been successfully conducted, including high-resolution SAR processing. Precise data synchronisation is achieved in subsequent processing steps. Bistatic image shows better resolution, SNR and no range ambiguities, as well as a different perspective of the imaged scene.

\section{ACKNOWLEDGEMENTS}

Special thanks to Franz Kurz (DLR-IMF) for providing Kaufbeuren optical image.

\section{REFERENCES}

[1] M. Wendler, G. Krieger, R. Horn et al., Results of a Bistatic Airborne SAR Experiment, Proc. International Radar Symposium 2003, DGON, pp. 247-253, Dresden, Germany.

[2] J. Ender, I. Walterschied, A. Brenner, New Aspects of Bistatic SAR: Processing and Experiments, Proc. IGARSS, 2004, Anchorage, USA.

[3] S. V. Baumgartner, M. Rodriguez-Cassola, A. Nottensteiner, Ralf Horn, Marco Schwerdt, Rolf Scheiber, Ulrich Steinbrecher, Robert Metzig, Markus Limbach, Josef Mittermayer, G. Krieger, A. Moreira, Bistatic Experiment Using TerraSAR-X and DLR's new F-SAR System, Proc. EUSAR 2008, Friedrichshafen, Germany.

[4] G. Krieger, M. Younis, Impact of Oscillator Noise in Bistatic and Multistatic SAR, IEEE GRSL 3-3, 2006.

[5] G. Krieger, H. Fiedler, D. Hounam, A. Moreira, Analysis of system concepts for bi- and multi-static SAR missions, Proceeding of IGARSS, 2003, Toulouse, France. 